\title{
Unified Universal Quantum Cloning Machine and Fidelities
}

\author{
Yi-Nan Wang ${ }^{1}$, Han-Duo Shi ${ }^{1}$, Zhao-Xi Xiong ${ }^{1}$, Li Jing ${ }^{1}$, Xi-Jun Ren ${ }^{2}$, Liang-Zhu Mu ${ }^{1 *}$, and Heng Fan $^{3 \dagger}$ \\ ${ }^{1}$ School of Physics, Peking University, Beijing 100871, China \\ ${ }^{2}$ School of Physics and Electronics, Henan University, Kaifeng 4750011, China \\ ${ }^{3}$ Institute of Physics, Chinese Academy of Sciences, Beijing 100190, China
}

(Dated: November 4, 2018)

\begin{abstract}
We present a unified universal quantum cloning machine, which combines several different existing universal cloning machines together including the asymmetric case. In this unified framework, the identical pure states are projected equally into each copy initially constituted by input and one half of the maximally entangled states. We show explicitly that the output states of those universal cloning machines are the same. One importance of this unified cloning machine is that the cloning procession is always the symmetric projection which reduces dramatically the difficulties for implementation. Also it is found that this unified cloning machine can be directly modified to the general asymmetric case. Besides the global fidelity and the single-copy fidelity, we also present all possible arbitrarycopy fidelities.
\end{abstract}

PACS numbers: 03.67.Ac, 03.65.Aa, 03.67.Lx, 03.65.Ta

Introduction.- No-cloning theorem is fundamental for quantum mechanics and quantum information science that states an unknown quantum state can not be cloned perfectly[1]. However, we can try to clone a quantum state approximately with the optimal quality [2], or instead, we can try to clone it perfectly with the largest probability 3]. So various quantum cloning machines have been designed for different quantum information tasks [4-18]. Experimentally, quantum cloning machines have been realized in optics system [19 22], nuclear magnetic resonance system [23, 24], diamond nitrogenvacancy center system [25], etc.

The universal quantum cloning machine is first proposed by Bužek and Hillery [2] which can copy optimally one arbitrary qubit equally well to two copies. Later more general cases have been studied, see Ref. 17] for a review. So far there exists two universal quantum cloning machines which can clone $N$ identical $d$-level pure quantum states to $M$ copies, $M \geq N$. One is proposed by Werner in Ref. [5], and the other is proposed by Fan et al. in Ref. [7]. Both have advantages from different points of view. Also we know some limited cases of asymmetric cloning [14]. It seems that all those cloning machines are quite different and no simple connection exists. In this Letter, we will present a simple and unified cloning transformation which combines all those cloning machines together. In the framework of the unified quantum cloning, the identical pure states are projected equally into each copy where the output is initially constituted by input and one half of the maximally entangled states, the left half of the maximally entangled states act as the ancillary states. We will show explicitly that the density operators from those cloning machines are the same. Since the symmet-

\footnotetext{
*muliangzhu@pku.edu.cn

†hfan@iphy.ac.cn
}

ric operator is constituted by SWAP gates, the quantum circuit corresponding to this unified cloning machine can thus be designed accordingly. Also importantly, this unified universal cloning machine can be easily modified to the general asymmetric case. As we know, only a few limited results of asymmetric cloning are known while the general case is still absent.

The optimality of the cloning machine is judged generally by whether the obtained fidelity of the cloning output state achieves its optimal bound. So far the optimal global fidelity and single-copy fidelity have already been obtained [5, 13]. It is, however, necessary to find optimal general-copy fidelities since it is possible that different optimal fidelities give different criteria, as happened in phase-covariant cloning machine [26]. With the unified and optimal universal cloning machine, we will present all possible arbitrary-copy fidelities so that the optimality of the cloning machine can be quantified from different aspects.

Equivalence of two universal quantum cloning machines.-For a pure state, $|\varphi\rangle=\sum_{j} x_{j}|j\rangle$, in $d$ dimensional Hilbert space $\mathcal{H}$ with density operator $\sigma \equiv|\varphi\rangle\left\langle\left.\varphi\left|, d=\operatorname{dim} \mathcal{H}, \sum_{j}\right| x_{j}\right|^{2}=1\right.$, we refer it to a qudit. The $N \rightarrow M$ Werner cloning machine is presented as [5],

$$
\rho^{\text {out }}=\frac{d[N]}{d[M]} s_{M}\left(\sigma^{N} \otimes \mathbb{I}^{\otimes(M-N)}\right) s_{M},
$$

where $d[N]=C_{d+N-1}^{N}=\frac{(d+N-1) !}{N !(d-1) !}$, it is the dimension of the symmetric subspace of $N$-fold Hilbert space, $d[N]=$ $\operatorname{dim} \mathcal{H}_{+}^{\otimes N}, \mathbb{I}$ is the identity on $\mathcal{H}, s_{M}$ is the symmetric projector which maps states in $\mathcal{H}^{\otimes M}$ onto its symmetric subspace $\mathcal{H}_{+}^{\otimes M}$. Explicitly, $s_{M}=\sum_{\vec{m}}^{M}|\vec{m}\rangle\langle\vec{m}|$, where state $|\vec{m}\rangle \equiv\left|m_{1}, m_{2}, \ldots, m_{d}\right\rangle$ is a completely symmetric state with $m_{j}$ states in $|j\rangle$, and the summation is assumed to run all possible values with constraint, $\sum_{j} m_{j}=M$.

In Werner cloning machine, the information of the in- 
put is equally projected to each copy. The initial $M-N$ identities are also understandable since the assumption of the universal cloning machine is that the input state is arbitrary thus a completely mixed state, $\mathbb{I} / d$, should be a suitable candidate before the cloning procession is applied.

Following Bužek-Hillery $1 \rightarrow 2$ universal cloning machine for qubit [2] and qudit [6], the $N \rightarrow M$ for qubit by Gisin and Massar [4], Fan et al. proposed the following transformation for qudit case [7], see Fig.1,

$$
U|\vec{n}\rangle \otimes R=\eta \sum_{\vec{k}}^{M-N} \sqrt{\prod_{j} \frac{\left(n_{j}+k_{j}\right) !}{n_{j} ! k_{j} !}}|\vec{n}+\vec{k}\rangle \otimes R_{\vec{k}},
$$

where $|\vec{n}\rangle$ is the input state, $U$ denotes unitary transformation, $R$ in l.h.s. denotes blank state and the initial ancillary state, $R_{\vec{k}}$ is the ancillary state which can be realized also by symmetric state $|\vec{k}\rangle$, the whole normalization factor takes the form, $\eta=$ $\sqrt{(M-N) !(N+d-1) ! /(M+d-1) !}$. Here, the summation is also assumed to run all possible values under the constraint $\sum k_{j}=M-N$. For $N$ identical pure states $|\varphi\rangle$, the input takes the form,

$$
|\varphi\rangle^{\otimes N}=\sqrt{N !} \sum_{\vec{n}}^{N} \prod_{j} \frac{x_{j}^{n_{j}}}{\sqrt{n_{j} !}}|\vec{n}\rangle,
$$

we can apply Eq. (2), trace out the ancillary states and thus obtain the output density operator. Similarly as for qubit case in theory [8] and in experiment [19], this cloning machine might naturally be realized by light emission from multilevel atomic system [9].

Typically, two different figures of merit are applied for the universal cloning machines. One is the global fidelity between the whole output density operator $\rho^{\text {out }}$ and the ideal output $|\varphi\rangle^{\otimes M}$ with perfect $M$ copies, $F_{M}={ }^{\otimes M}\left\langle\varphi\left|\rho^{\text {out }}\right| \varphi\right\rangle^{\otimes M}$. The other is the single-copy fidelity defined between an individual output density operator and a single input pure state, $F_{1}=\left\langle\varphi\left|\rho_{1}^{\text {out }}\right| \varphi\right\rangle$, where each individual output density operator $\rho_{1}^{\text {out }}$ is the same for all $M$ copies. It is shown that those two fidelities for Werner cloning machine and for cloning machine in (2) are the same. Yet the similarity of the output density operators is necessary. Next, explicitly we shall show the output state of $M$ copies from the two cloning machines are the same.

First, we can find that the symmetric state $|\vec{m}\rangle$ of $M$ qudits can be divided into two parts with $\mathrm{N}$ qudits and $M-N$ qudits, respectively,

$$
|\vec{m}\rangle=\frac{1}{\sqrt{C_{M}^{N}}} \sum_{\vec{k}}^{M-N} \prod_{j} \sqrt{\frac{m_{j} !}{\left(m_{j}-k_{j}\right) ! k_{j} !}}|\vec{m}-\vec{k}\rangle|\vec{k}\rangle .
$$

The symmetric projector $s_{M}$ can thus be reformulated by this splitting, then with the help of the expansion

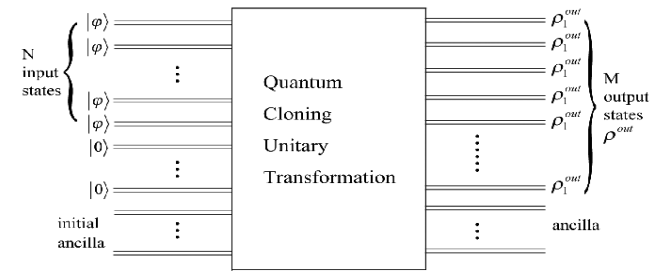

FIG. 1: Generally, the input of the cloning machines are identical pure input states, blank states and initial ancilla, the cloning is realized by unitary transformation.

(3), substituting $\sigma^{\otimes N}$ into (11), by some calculations, the output density operator of Werner cloning machine takes the form,

$$
\begin{aligned}
\rho^{\text {out }}= & N ! \eta^{2} \sum_{\vec{m}, \vec{m}^{\prime}}^{M}|\vec{m}\rangle\left\langle\vec{m}^{\prime}\right| \\
& \times\left(\sum_{\vec{k}}^{M-N} \prod_{j} \frac{x_{j}^{m_{j}-k_{j}} x^{*\left(m_{j}^{\prime}-k_{j}\right)} \sqrt{m_{j} ! m_{j}^{\prime} !}}{\left(m_{j}-k_{j}\right) !\left(m_{j}^{\prime}-k_{j}\right) ! k_{j} !}\right)
\end{aligned}
$$

where we have already used $\left\langle\vec{l} \mid \overrightarrow{l^{\prime}}\right\rangle=\delta_{\overrightarrow{l^{\prime}}}$.

For the second cloning machine (2), substituting the result of (3) into (2) and tracing out the ancillary states, simply the output state is written as

$$
\begin{aligned}
\rho^{\prime \text { out }}= & N ! \eta^{2} \sum_{\vec{n}, \vec{n}^{\prime}}^{N} \sum_{\vec{k}}^{M-N}|\vec{n}+\vec{k}\rangle\left\langle\vec{n}^{\prime}+\vec{k}\right| \\
& \times\left(\prod_{j} \frac{x_{j}^{n_{j}} x_{j}^{*\left(n_{j}^{\prime}\right)} \sqrt{\left(n_{j}+k_{j}\right) !\left(n_{j}^{\prime}+k_{j}\right) !}}{n_{j} ! n_{j}^{\prime} ! k_{j} !}\right)
\end{aligned}
$$

Considering we have the constraint $\sum k_{j}=M-N$, apparently, the output states from two universal quantum cloning machines are the same, $\rho^{\text {out }}=\rho^{\prime \text { out }}$.

Unified universal quantum cloning machine.Stimulated by the fact that the two existing universal cloning machines are the same, we may try further to explore the possibility to unify the two cloning machines together. For $N$ identical pure states, we propose the $N \rightarrow M$ universal cloning machine as the following,

$$
|\varphi\rangle^{\text {out }}=\lambda\left(s_{M} \otimes \mathbb{I}^{\otimes(M-N)}\right)|\varphi\rangle^{\otimes N}\left|\Phi^{+}\right\rangle^{\otimes(M-N)},
$$

where $\left|\Phi^{+}\right\rangle \equiv \frac{1}{\sqrt{d}} \sum_{j}|j j\rangle$ is a maximally entangled state in $\mathcal{H} \otimes \mathcal{H}, \lambda$ is the normalization factor. Here operator $s_{M}$ acts on the $N$ identical pure input states and half of the $M-N$ maximally entangled states, the left half of the maximally entangled states are the ancillary states, see Fig.2.

Next we will show that this cloning transformation is the same as (2). We consider a property of the symmetric 


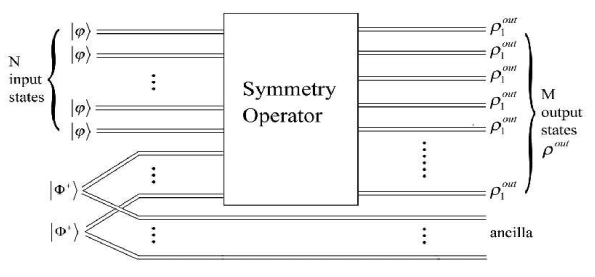

FIG. 2: The unified cloning machine is constituted by identical pure input states and the prepared maximally entangled states, the cloning is always realized by symmetric projection.

projector, $s_{M}=s_{M}\left(\mathbb{I}^{\otimes N} \otimes s_{M-N}\right)$, this is due to Eq.(4) and also $s_{M-N}$ is the identity operator on $\mathcal{H}_{+}^{\otimes(M-N)}$. By symmetric projection, $M-N$ maximally entangled states can be mapped as a maximally entangled state in symmetric subspace $\mathcal{H}_{+}^{\otimes(M-N)} \otimes \mathcal{H}_{+}^{\otimes(M-N)}$,

$$
\left(s_{M-N} \otimes \mathbb{I}^{\otimes(M-N)}\right)\left|\Phi^{+}\right\rangle^{\otimes(M-N)}=\sum_{\vec{k}}^{M-N}|\vec{k}\rangle|\vec{k}\rangle,
$$

where an unimportant whole factor is omitted, and some other unimportant whole factors will also be omitted later without specification. Note that the entanglement cutting is unchanged here. Since quantum mechanics is linear, so we just consider the input state be a symmetric state for (77), with the help of the result in (8), we can find the unified cloning machine (7) can be rewritten as the form

$$
\begin{aligned}
& \left(s_{M} \otimes \mathbb{I}^{\otimes(M-N)}\right)|\vec{n}\rangle\left|\Phi^{+}\right\rangle^{\otimes(M-N)} \\
& =\left(s_{M} \otimes \mathbb{I}^{\otimes(M-N)}\right)|\vec{n}\rangle \sum_{\vec{k}}^{M-N}|\vec{k}\rangle|\vec{k}\rangle \\
& =\sum_{\vec{k}}^{M-N} \sqrt{\prod_{j} \frac{\left(n_{j}+k_{j}\right) !}{n_{j} ! k_{j} !}}|\vec{n}+\vec{k}\rangle|\vec{k}\rangle,
\end{aligned}
$$

where the splitting relation (44) is used in the last equation. Thus considering that the last $M-N$ qudits are ancillary states, the universal cloning transformation (2) is re-obtained by the unified cloning transformation (7). Also (7) can be considered to be an equivalent form of Werner cloning machine since by taking trace over the last $M-N$ qudits where each maximally entangled state will provide an identity on $\mathcal{H}$, we will re-obtain Werner cloning machine (11).

We remark that the unified cloning machine (7) can be easily understood, which is a property inherited from Werner cloning machine, and it has also the explicit transformations as those in, such as Refs. [2, [4, 6, 7].

General fidelities.-The merit of the cloning machine is generally quantified by fidelity between input and output, the global fidelity and single-copy fidelity are presently known. As we mentioned, it is also necessary to have all possible arbitrary-copy fidelities to offer a full description of the merit of universal cloning machine. With output density operator $\rho^{\text {out }}$ available, we can find the reduced density operators of $L$ qudits, $\rho_{L}^{\text {out }}$, where $1 \leq L \leq M$. The general fidelities are defined as $F_{L} \equiv{ }^{\otimes L}\left\langle\varphi\left|\rho_{L}^{\text {out }}\right| \varphi\right\rangle^{\otimes L}$. We remark that all reduced density operators of $L$ qudits at different positions are the same which is ensured by the fact that the output is in symmetric subspace $\mathcal{H}_{+}^{\otimes M}$. By straightforward but tedious calculations, we find,

$$
\begin{aligned}
& F_{L}=\frac{(d+N-1) !(M-N) !(M-L) !}{(d+M-1) ! M ! N !} \times \\
& \sum_{m_{1}} \frac{\left(M-m_{1}+d-2\right) !\left(m_{1} !\right)^{2}}{\left(m_{1}-L\right) !\left(m_{1}-N\right) !(d-2) !\left(M-m_{1}\right) !}
\end{aligned}
$$

where $m_{1}$ is one entry of the vector $\vec{m}$, here we have considered the property that the output state $\rho^{\text {out }}$ is covariant for the cloning transformation, i.e., $\rho^{\text {out }}$ is changed as $u^{\otimes M} \rho^{\text {out }} u^{\dagger \otimes M}$ when $|\varphi\rangle$ is changed as $u|\varphi\rangle$ [5].

For $L=1, L=M$, we recover the known results [5, 13]. $F_{M}=d[N] / d[M]$, and $F_{1}=$ $(N(d+M)+M-N) /(d+N) M$.

For special case $N=1$, the general fidelity (10) can be simplified as,

$$
F_{L}(N=1)=\frac{L ! d ![L(d+M)+M-L]}{(d+L) ! M} .
$$

Here we have used the identity:

$$
\begin{aligned}
& \frac{(M-N) !(N+d-1) !}{(M+d-1) ! N !} \times \\
& \sum_{m=0}^{M-N} \frac{((N+m) !)^{2}(M-N-m+d-2) !}{M m(M-N-m) !(d-2) !} \\
& =\frac{N(d+M)+M-N}{(d+N) M}
\end{aligned}
$$

The proof of this identity is mainly based on a permutation and combination equation [28].

Extension of the unified cloning machine to asymmetric case and examples.-As an example, let us consider the 1 to 2 cloning machine for qudit and qubit. The symmetric projector takes the form $s_{2}=\sum|j j\rangle\langle j j|+$ $\frac{1}{2} \sum_{j \neq l}(|j l\rangle+|l j\rangle)(\langle j l|+\langle l j|)$. Up to a whole factor, the unified cloning machine can be written as,

$$
\begin{aligned}
& \left(s_{2} \otimes \mathbb{I}\right)|l\rangle_{1}\left|\Phi^{+}\right\rangle_{2 a}=|l l\rangle_{12}|l\rangle_{a} \\
& +\frac{1}{2} \sum_{j \neq i}\left(|l j\rangle_{12}+|j l\rangle_{12}\right)|j\rangle_{a},
\end{aligned}
$$

where states with subindex $a$ is the ancilla. Really this is the optimal universal cloning machine presented in 
Ref.[6]. For qubit case, we have the well known BužekHillery cloning machine,

$$
\begin{aligned}
& |0\rangle \rightarrow \sqrt{\frac{2}{3}}|00\rangle|0\rangle_{a}+\sqrt{\frac{1}{6}}\left(|01\rangle_{12}+|10\rangle\right)|1\rangle_{a}, \\
& |1\rangle \rightarrow \sqrt{\frac{2}{3}}|11\rangle|0\rangle_{a}+\sqrt{\frac{1}{6}}\left(|01\rangle_{12}+|10\rangle\right)|0\rangle_{a} .
\end{aligned}
$$

As we already know, besides the case of symmetric output, we can adjust the qualities of the individual output states in an imbalanced way. This is realized by the asymmetric cloning machine [14]. For 1 to 2 unified cloning machine, where projection $s_{2}$ is used, we know that, $s_{2}$ can be written as a summation of identity and a permutation, $s_{2}=\frac{1}{2}\left(\mathbb{I}^{\otimes 2}+\mathcal{P}\right)$, where $\mathcal{P}$ is the permutation (SWAP) operator, $\mathcal{P}|j l\rangle=|l j\rangle$. We can then consider to adjust the weights of identity and permutation in an imbalanced way. Naturally, we can change symmetric projector to asymmetric case as, $s_{2} \rightarrow \alpha \mathbb{I}^{\otimes 2}+\beta \mathcal{P}$, where $\alpha$ and $\beta$ are weights for adjusting. The corresponding asymmetric unified cloning machine is now changing as,

$$
|\varphi\rangle \rightarrow \alpha|\varphi\rangle_{1}\left|\Phi^{+}\right\rangle_{2 a}+\beta|\varphi\rangle_{2}\left|\Phi^{+}\right\rangle_{1 a}
$$

note the orders of the subindices in these two terms are different, also those two terms are not orthogonal. The problem now is whether this cloning procession is optimal. We know that, $|\varphi\rangle_{2}\left|\Phi^{+}\right\rangle_{1 a}=\frac{1}{d} \sum\left(U_{j l}|\varphi\rangle_{1}\right)\left|\Phi_{j l}\right\rangle_{2 a}$, where $U_{j l}$ are generalized Pauli matrices and identity, $\left|\Phi_{j l}\right\rangle_{2 a}$ are orthonormal maximally entangled states with $\left|\Phi_{00}\right\rangle=\left|\Phi^{+}\right\rangle$. Now exactly, we find that (13) is the optimal asymmetric cloning proposed by Cerf [14, 15].

So far only limited cases of the asymmetric cloning machine have been presented [14 16]. The general asymmetric cloning is still absent possibly because that the formulae are too complicated to be extended. Here similar as for the case of 1 to 2, the unified cloning machine can be adjusted to the general asymmetric cloning machine and the related entanglement sharing inequalities [27]. The method is to plug into a weight for each essential permutation to modify the symmetric operator $s_{M}$ in (77), the problem is like to put $N$ balls into $M$ boxes with a weight for each choice. Thus we offer a simple realization of the asymmetric cloning. When all weights are equal, it reduces to the symmetric case.

Conclusions. - We present a unified optimal universal cloning machine. The cloning procession is equivalent with Werner cloning machine [5] and the one proposed by Fan et al. [7] and can be easily adjusted to asymmetric cloning machines [14 16, 27] and to the general case. This simple cloning machine is always realized by a symmetric projection and initially prepared maximally entangled states and thus should reduce the difficulties for implementation. Also the general fidelities are obtained. Our result offers a new platform for other cloning tasks for cases like phase-covariant and state-dependent.

This work is supported by NSFC (10974247,
11047174), "973" program (2010CB922904) and NFFTBS (J1030310).

[1] W.K. Wootters and W. H. ZureK, Nature (London) 299 , 802(1982).

[2] V. Bužek and M. Hillery, Phys. Rev. A 54, 1844 (1996).

[3] L.M.Duan and G.C.Guo, Phys.Rev.Lett.80,4999(1998).

[4] N. Gisin and S. Massar, Phy. Rev. Lett. 79, 2153 (1997).

[5] R. F. Werner, Phy. Rev. A 58, 1827 (1998).

[6] V.Bužek and M.Hillery, Phys.Rev.Lett. 81, 5003 (1998).

[7] H. Fan, K. Matsumoto, and M. Wadati, Phys. Rev. A 64, 064301 (2001).

[8] C. Simon, G. Weihs, and A. Zeilinger, Phys. Rev. Lett. 84, 2993 (2000).

[9] H. Fan, G. Weihs, K. Matsumoto, and H. Imai, Phys. Rev. A 66, 024307 (2002).

[10] D. Bruß, D. DiVincenzo, A. Ekert, C. A. Fuchs, C. Macchiavello, and J. A. Smolin, Phys. Rev. A 57, 2368 (1998).

[11] N. Gisin and S. Massar, Phys. Rev. Lett. 79, 2153 (1997).

[12] D. Bruß, A. Ekert, and C. Macchiavello, Phys. Rev. Lett. 81, 2598 (1998).

[13] M. Keyl and R. F. Werner, J. Math. Phys. 40, 3283 (1999).

[14] N. J. Cerf, Phys. Rev. Lett. 84, 4497 (2000).

[15] N. J. Cerf, J. Mod. Opt. 47, 187 (2000).

[16] J. Fiurasek, R. Filip, J. J. Cerf, Quant. Inform. Comp. 5, 583 (2005).

[17] V. Scarani, S. Iblisdir, N. Gisin, and A. Acin, Rev. Mod. Phys. 77, 1225 (2005).

[18] H. Fan, K. Matsumoto, X. B. Wang, and M. Wadati, Phys. Rev. A 65, 012304 (2002).

[19] A. Lamas-Linares, C. Simon, J. C. Howell, and D. Bouwmeester, Science 296, 712 (2002).

[20] E. Nagali et al., Nature Photonics 3, 720 (2009).

[21] F.Sciarrino, F.De Martini, Phys. Rev. A 72, 062313 (2005).

[22] C. Vitelli et al. Phys. Rev. Lett. 105, 113602 (2010).

[23] J. F. Du et al. Phys. Rev. Lett. 94, 040505 (2005)

[24] H. Chen, X. Zhou, D. Suter, and J. F. Du, Phys. Rev. A 75, 012317 (2007).

[25] X. Y. Pan, G. Q. Liu, L. L. Yang, and H. Fan, arXiv:1009.2618

[26] H. Fan, K. Matsumoto, X. B. Wang, and H. Imai, J. Phys. A 35, 7415 (2002).

[27] A. Kay, D. Kaszlikowski, and R. Ramanathan, Phys. Rev. Lett. 103, 050501 (2009).

[28] The equation can be written as,

$$
\begin{aligned}
& \sum_{m=0}^{M-N}\left(\frac{(N+m) !}{N ! m !}\right)\left(\frac{(M-N-m+d-2) !}{(M-N-m) !(d-2) !}\right)(N+m) \\
= & N \sum_{m=0}^{M-N} C_{N+m}^{N} C_{M-N-m+d-2}^{d-2}+ \\
& +(N+1) \sum_{m=0}^{M-N} C_{N+m}^{N+1} C_{M-N-m+d-2}^{d-2} \\
= & N \cdot C_{M+d-1}^{M-N}+(N+1) C_{M+d-1}^{M-N-1} .
\end{aligned}
$$

